\title{
TRABAJO DE DOCENTE RURAL Y APLICA- CIÓN DE INGENIERÍA Y TECNOLOGÍAS DE INFORMACIÓN EN EL ÁMBITO LABORAL
}

Lic. Alicia Amarilla, Ing. Norberto Sanabria, Ing. Armando Achem, Lic. Alejandro Berdaguez, Bioing. Hernán Fernández Céspedes $\left({ }^{*}\right)$

\section{Resumen}

El objetivo de ésta investigación fue analizar las condiciones de trabajo de los docentes que ejercen la profesión en el nivel inicial y en los primeros años del ciclo primario, en escuelas rurales de la provincia de Corrientes, su relación con la satisfacción laboral y el impacto sobre ellas de la aplicación de las TIC, como la Telemedicina.

A partir del estudio se detectaron factores que afectan de manera negativa y otros de manera positiva a la satisfacción de éstos docentes en su ámbito de trabajo.

Con los resultados obtenidos se buscó la aplicación de tecnologías de información y las ingenierías en sus disciplinas de telecomunicaciones y bioingeniería para mejorar la calidad de vida de la población involucrada, optimizando los recursos existentes en los establecimientos educativos.

Palabras Clave: Satisfacción laboral, Condiciones y Medio Ambiente de Trabajo, Tecnologías de Información y Comunicación, Telemedicina.

\section{Abstract}

The purpose of this study was to analyze the working conditions of teachers practicing in the initial and in the early years of primary education in rural schools in the province of Corrientes and its relationship to job satisfaction.

From the study factors affecting negatively and others positively to the satisfaction of these teachers in their field of work so detected.

With the results of the application of information technology and engineering disciplines in their telecommunications and bioengineering sought to improve the quality of life of the population involved, optimizing existing resources in educational establishments.

Keywords: Job satisfaction, Conditions and Working Environment, Information and Communication Technologies, Telemedicine.

\section{Introducción}

La importancia de la actividad docente, la complejidad del nivel inicial y de los primeros años del ciclo primario y la diversidad de condiciones socio-culturales y económicas que el docente rural enfrenta; nos llevó a pensar tanto, en su satisfacción laboral como en las condiciones de trabajo en las que desarrollan su labor. Con los antecedentes de investigación en esta temática para esta población ${ }^{1}$, se buscó explorar desde la perspectiva del aprovechamiento de 
las Tecnologías de Información y la Electrónica en su especialidad biomédica.

El trabajo produce efectos sobre el plano físico, el mental y el afectivo o psíquico; esto genera riesgos psicosociales. Sin embargo el trabajo no es un patógeno, si no lo son las condiciones del mismo.

Es necesario también tener en cuenta que cada trabajador es un sujeto, no un recurso. El término RRHH hoy resulta retrógrado.

El trabajo docente es uno de los que generan mayores riesgos psicosociales.

Según Hans Selye en su tratado "Estrés de la Vida" el Síndrome General de Adaptación (SGA) deviene de estresantes, que son agentes nocivos, procesos emocionales, mentales o fisiológicos diversos, percibidos como amenazantes y producen distintas respuestas en las personas.

Neffa, Julio Cesar (2002) hace referencia al trabajo docente en el marco de la concepción renovadora y concluye que la tarea docente de escuelas primarias constituye una actividad laboral en el sentido estricto del concepto, que comprende todas las dimensiones de las personas, de los trabajadores y de la educación.

En general, los docentes rurales tienen que enfrentar y proponer soluciones a diversos conflictos. A modo de ejemplo, se mencionan algunos: conflictos socio-culturales y/o familiares de los estudiantes; condiciones edilicias precarias (escasa iluminación, espacios inapropiados y mobiliario deficiente) e inconvenientes en el servicio de transporte, escasos medios de comunicación, muy baja posibilidad de atención de urgencias sanitarias de los docentes y alum- nos, entre otros.

La población seleccionada para la investigación recorre varios kilómetros para llegar a los establecimientos educativos, utilizando medios convencionales cuando las condiciones climáticas son favorables; pero cuando éstas no resultan óptimas, deben optar por transportes alternativos (caballos, canoas, etc.), lo que demanda mayor esfuerzo, tiempo y dinero.

Al mismo tiempo, el aislamiento de algunas poblaciones y las dificultades en el desplazamiento de sus habitantes, las distintas situaciones socioeconómicas mayormente desfavorables, la tecnificación de las tareas rurales y la consiguiente disminución de fuentes de trabajo y el agotamiento de los recursos naturales, suscita que importantes sectores de la población alejada de centros urbanos se encuentren con necesidades básicas insatisfechas: educación, vivienda, saneamiento ambiental y servicios sanitarios.

Sumado a lo anterior, en la actividad de los docentes rurales se verifica una mayor complejidad debido, entre otros factores, a las características propias de la escolaridad en el medio rural y la escasa teorización de la experiencia didáctica que se desarrolla con la modalidad de Plurisalas (alumnos de distintos grados comparten el mismo espacio físico, horario y docente para el aprendizaje).

En este contexto, los docentes ejercen la profesión ante niños de diversas edades, provenientes de familias con distintos grados de instrucción y diferentes condiciones socio-económicas.

La relevancia de la actividad docente,

1. "Estudio de las condiciones de trabajo y la satisfacción laboral en docentes rurales de la provincia de Corrientes". Amarilla, Alicia. e-mail: amarillaalicia@hotmail.com 
las condiciones laborales anteriormente expuestas, el escaso aprovechamiento de las tecnologías informáticas y las telecomunicaciones para satisfacer la demanda del sector y la falta de profundización en la investigación en el área para este grupo de trabajadores, motivaron el trabajo.

Con el trabajo de investigación se ha constatado que uno de problemas existentes en la lejanía de los establecimientos escolares, los escasos de medios de transporte para el traslado de compañeros docentes o de alumnos en el caso de que suceda alguna emergencia médica.

Para analizar algunos puntos que nos interesa del trabajo docente, tomamos como referencia la bibliografía de Martínez, Deolidia (1990) "El riesgo de Enseñar", quien manifiesta que el maestro tiene que ir construyendo defensas propias para así lograr sobrevivir en su trabajo cotidiano y, además, debe asumir consciente o inconscientemente la unión de lo que esta desunido, es decir, tiene que resolver de alguna manera los problemas causados por la desarticulación del trabajo y la pedagogía con el contenido de las materias que enseña y las condiciones y medio ambiente de trabajo. Todo esto genera en el trabajador docente una fatiga especial que lo convierte poco a poco en un ser defensivo, bloqueado, improductivo e insatisfecho.

Martínez (1990) expresa: "No se necesita muchas estadística para probar que la salud del sector laboral está bastante deteriorado a nivel mundial. Basta con hacer un recuento de las licencias médicas otorgadas a los maestros por problemas psiquiátricos (más del 70\% en España y 60\% en Argentina). El docente no se permite a si mismo dejar de trabajar ya que, en primer lugar, siente que sería como dejar a los niños en abandono, por factores económicos e ideológicos, además de la fuerte censura grupal frente al que "afloja", los compañeros de trabajo ejercen una dura crítica contra los que faltan".

Continuando con el análisis de Martínez (1990), la autora hace referencia a que el trabajo docente, comparado con otros trabajos, presenta características de franco aislamiento que genera una patología que, en mayor o menor grado, perturba la vida social y privada del trabajador. Este aislamiento comienza a superarse cuando hay posibilidades de compartir el trabajo cotidiano dentro del tiempo asignado a la jornada laboral. Por ello, un buen principio para eliminar las causas del aislamiento es transformar el sistema de organización del trabajo docente en escuelas.

\section{Conceptos básicos}

\section{Condiciones y Medio Ambiente de Trabajo}

Las Condiciones y Medio Ambiente de Trabajo (CyMAT) están constituidas por los factores socio-técnicos y organizacionales del proceso de producción implementado en el establecimiento (o condiciones de trabajo) y por los factores de riesgos del medio ambiente de trabajo. Ambos grupos de factores constituyen las exigencias, requerimientos y limitaciones del puesto de trabajo cuya articulación sinérgica da lugar a la carga global del trabajo prescripto, el cual es asumido, asignado o impuesto a cada trabajador, provocando de manera mediata o inmediata, efectos directos o indirectos, positivos o negativos sobre la salud física, psíquica y/o mental de los trabajadores (Neffa, 1988).

Además, sostiene que los trabajadores deben ser reconocidos como seres individuales y no como sujetos homogéneos y que, además, el proceso de trabajo comien- 
za a tomarse en su aspecto micro y macro, entendiendo que las CyMAT están relacionadas con las condiciones que hacen a la carga física, a la seguridad en él y a la carga psíquica y mental del trabajador.

Tomando como punto de partida la concepción renovadora descripta, se considera que son múltiples los factores que contribuyen a diferenciar entre sí a los miembros de un colectivo de trabajo, entre ellos la edad, el sexo, el origen socio-profesional y el medio geográfico e histórico en el cual nacieron y se desarrollaron. La capacidad de adaptación y de resistencia a los factores de riesgos son muy diferentes según sean las personas, es también diferente su percepción y su vivencia de trabajo y los factores de riesgos inherente al mismo. La heterogeneidad tiene, incluso, raíces más profundas si se analiza desde la perspectiva de las condiciones y medio ambiente de trabajo. En virtud a esta heterogeneidad que prima dentro de la fuerza laboral, debe relativizarse la utilidad y la aplicabilidad de las nociones tales como máximas admisibles de concentración, valores límites de exposición y tiempo máximo permisible de exposición a riesgos.

Los factores socio-técnicos y organizacionales del proceso de producción que están presente en el establecimiento, van a jugar un papel decisivo, aunque no exclusivo, para especificar las condiciones de trabajo vigente.

Figura 1: Taller a docentes

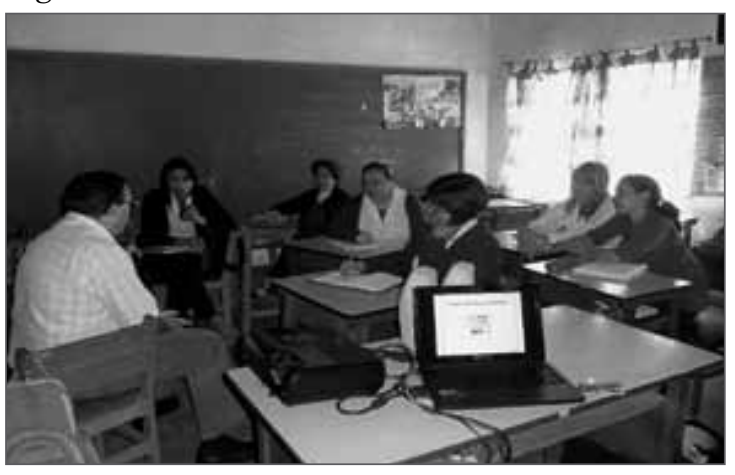

En cuanto a la organización y el contenido de trabajo, estos factores están determinados directamente por el tipo de proceso de trabajo que predomine en la empresa y particularmente en el puesto de trabajo.

La organización del trabajo se relaciona estrechamente con las dimensiones cognitivas de los trabajadores, dando lugar a la carga mental.

El contenido del trabajo al nivel del puesto, se articula directamente con la división social y técnica del trabajo.

\section{Riesgos Latentes}

Rosskam (1997), Nieto (1999) y Romera (2001) indican que los riesgos físicos, químicos, biológicos y ergonómicos y la relación de éstos con la satisfacción laboral están estrechamente relacionados y observan que un trabajador insatisfecho tiene mayor probabilidad de sufrir accidentes. Por su parte, Adams (2000) y Romera (2001) en investigaciones sobre riesgos ergonómicos, consideran que se debería medir conjuntamente el grado de satisfacción laboral con la exposición a diversos factores de riesgo y sugieren que en los riesgos ergonómicos influyen tanto en los aspectos físicos como los psicosociales; tales como el descontento en el trabajo, la monotonía, control limitado del trabajo y alta exigencia mental.

La Organización Mundial de la Salud, estima que en América Latina y el Caribe sólo se notifican entre el 1\% y el 5\% de enfermedades ocupacionales, (OPS 2003). En la Argentina, la Superintendencia de Riesgos del Trabajo y las Aseguradoras de Riesgos de Trabajo (ART), consideran que un ambiente de trabajo sano y seguro es un factor de productividad y competitividad, y los 
lineamientos estratégicos de estas entidades proponen que se deben eliminar o controlar los riesgos laborales mediante proyectos institucionales que incluyan líneas de investigación al respecto (Verón, 2003).

\section{Telemedicina}

Dentro de la ingeniería biomédica se promueve el cuidado de pacientes mediante la aplicación de la ingeniería y los conocimientos de gestión a la tecnología sanitaria.

Si bien esta es una tarea difícil para los ingenieros, en los últimos años ha habido avances importantes para acercar los servicios a la sociedad más vulnerable y de bajos recursos, en especial a los habitantes de lugares más alejados de hospitales públicos o centros de atención primaria.

La telemedicina busca la atención clínica de pacientes mediante la utilización de medios remotos y el aprovechamiento de las TIC. El constante aumento de la demanda de la sociedad de servicios asistenciales, obliga inexorablemente a los ingenieros a trabajar en grupos interdisciplinarios que diseñen medios más eficaces y rentabilizar al máximo los recursos disponibles.

La existencia de poblaciones escolares muy alejadas de los centros urbanos, en lugares remotos y de difícil accesibilidad y el escaso aprovechamiento de la tecnología para la atención de ciertas necesidades, como la atención sanitaria, deja a estos individuos en inferiores condiciones respecto a otros ciudadanos.

Los medios de transporte son limitados, por lo que los pobladores de estas zonas acuden muy poco a las ciudades; normalmente las distancias a cubrir desde las escuelas hasta algún centro de salud especializado se encuentra a varias horas de camino.

La situación descripta dificulta la detección, prevención y tratamientos de enfermedades en éstas poblaciones.

E1 desarrollo de sistemas con herramientas propias de la telemedicina provocaría una mejora en las condiciones sanitarias en estos establecimientos laborales.

La utilización de la informática y las nuevas tecnologías de transmisión de datos son de alta aplicabilidad en las tareas sanitarias, ya que se dispone de información sobre el pasado y el presente del paciente, incluidos datos sobre su entorno vital y casos similares. Esta definición incluye numerosos campos de aplicación que van desde la investigación a la asistencia o a la prestación de servicios a un usuario individual o a la comunidad. Como ventajas para los profesionales pueden distinguirse:

Mejor accesibilidad a los datos sanitarios del Usuario, con la posibilidad de una historia clínica informatizada, independientemente de su ubicación geográfica.

La teleformación o teledocencia con los protocolos de Internet hace posible una formación homogénea, de calidad, accesible y barata (Ávila de Tomás, 2001).

La telemedicina, basado en el uso de las consultas de vídeo interactivas, se está utilizando con mayor frecuencia en el medio rural. Este desarrollo es potencialmente importante para los pacientes rurales porque hay menos médicos especialistas. La disminución de los costos de los equipos de telemedicina y de la transmisión, han creado un mayor acceso a estas tecnologías para los médicos de familia rurales y sus pacientes (Norris y Thomas, 2002).

Por medio de la telemedicina la interac- 
ción entre el paciente y el médico trasciende las fronteras geográficas y temporales, evita desplazamientos innecesarios, acorta los tiempos de espera en la atención y permite el diagnóstico y tratamiento a distancia desde centros especializados, cubriendo el bache generado por la falta de recursos humanos (RRHH) calificados y recursos físicos necesarios en zonas rurales. Hoy en día, la telemedicina no debe entenderse simplemente como una tecnología, sino como un nuevo sistema organizativo de la profesión médica. En este sentido, su aplicación representa una nueva manera de hacer y organizar la provisión de servicios sanitarios en beneficio de los pacientes, de los profesionales médicos y del sistema sanitario en general. La utilización de la TM hace que las distancias y los tiempos entre la atención primaria y la hospitalaria se acorten (Carnirero y Fernández, 2012).

La confidencialidad de la información transmitida y recibida debe garantizarse a través de mecanismos de seguridad. Su ausencia constituye una violación de los principios de ética médica, salvo situaciones de peligro inminente para la vida (Barreiro Martins y Simões, 2012).

\section{Aplicación de las Tecnologías de Información}

Se deben generar las condiciones adecuadas para que los docentes y alumnos de establecimientos en condiciones de ruralidad sean tratados individualmente en su contexto social y económico. Resulta necesario proponer un mayor aprovechamiento de las herramientas TIC existentes en éstos ámbitos para desnaturalizar de la desigualdad existente.

\section{ABORDAJE METODOLÓGICO}

\section{Estrategia Metodológica}

En la investigación se propuso analizar la relación entre la satisfacción laboral y las condiciones de trabajo de los docentes que ejercen la profesión en el nivel inicial y en los primeros años del ciclo primario, en escuelas rurales de la Provincia de Corrientes, en su primera etapa.

Se realizó un estudio de carácter cualicuantitativo, de tipo descriptivo. Se buscó especificar las propiedades importantes de los diferentes fenómenos, que fueron sometidos a análisis (Dankhe, 1986). Identificamos aspectos emergentes del campo empírico, a partir de la visita a los establecimientos escolares rurales, en el cual los docentes, ejercen su tarea.

Se definieron una serie de categorías de variables a fin de abordar las condiciones de trabajo y de la satisfacción laboral de los docentes que ejercen su profesión en establecimientos rurales en el nivel inicial y el nivel primario de la provincia de Corrientes.

\section{Técnicas de Recolección}

El relevamiento permitió conocer, caracterizar, identificar y describir las condiciones del medio ambiente de trabajo, revelar potenciales riesgos y obtener información sobre fenómenos o acontecimientos tal y como se producen.

Los procedimientos utilizados para recabar la información fueron los siguientes:

OBSERVACION: Para el relevamiento de los datos del medio ambiente de trabajo, se procedió a completar una ficha diseñada con anterioridad, a través de la cual se pretendía obtener la descripción de las características relevantes del lugar de trabajo de 
los docentes como ser: existencia de agua potable, luz eléctrica; estado de los edificios e instalaciones (piso y paredes de las aulas, ventilación e iluminación de las mismas); amoblamientos; distancia y tipo de caminos recorridos por los docentes para llegar a los lugares de trabajo (In Itinere).

Para conocer los aspectos inherentes a las condiciones de trabajo y a la satisfacción laboral de los docentes rurales, que poseían en el monto de la visita, se realizaron ENTREVISTAS ABIERTAS. Éstas permitieron recabar información sobre la problemática planteada e identificar las condiciones de trabajo y el estado de la satisfacción laboral.

Para la implementación del instrumento antes nombrado, se realizó una guía de preguntas semi-estructuradas a modo de orientación, la cual, debía ser completada en el mismo lugar de trabajo, durante la entrevista, y, además, permitió a los docentes entrevistados explayarse sobre un concepto propio del tema a tratar "la satisfacción laboral", con el objetivo de analizar todas las variables planteadas.

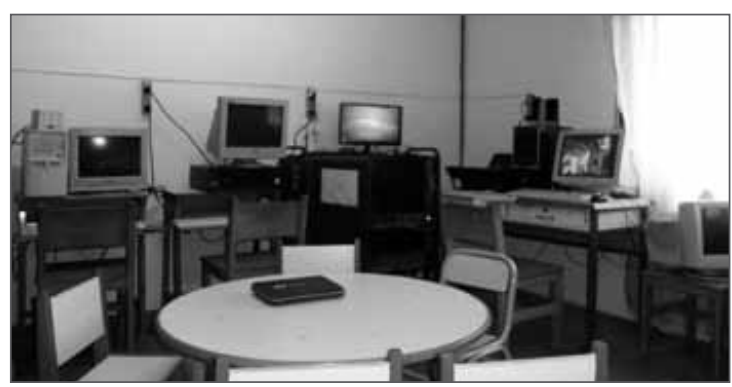

Figura 2. Relevamiento de equipos informáticos existentes en escuelas

\section{Muestra}

Para éste trabajo se tomaron como muestra 34 docentes que estuvieron dispuestos a colaborar con esta investigación, utilizando la técnica de muestra teórica, también de- nominado muestreo intencionado, mediante voluntarios y se realizó posteriormente un proceso de avalancha.

La cantidad de entrevistados seleccionados siguió el criterio de la Saturación Teórica (Glaser y Strauss, 1967).

Con el objeto de lograr que la muestra brinde la información necesaria para el estudio planteado sobre los docentes de la provincia, se realizaron las visitas a los establecimientos de diferentes puntos geográficos de Corrientes, tomando los departamentos de Paso de los Libres, Monte Caseros, San Martín, Bella Vista y Capital como referencias de zonas.

\section{Conclusiones}

Luego de haber realizado el análisis de las condiciones de trabajo a los que están expuestos los docentes rurales del nivel inicial y primeros años del ciclo primario de

la provincia de Corrientes, desde la perspectiva del trabajador y del significado que le da a la satisfacción laboral cada uno de ellos, estamos en condiciones de afirmar que existe una alta relación entre ambos conceptos y que el impacto de esta satisfacción está determinada por las condiciones de trabajo.

A través del análisis realizado, los docentes coinciden expresando conformidad en los siguientes aspectos: poseen libertad absoluta para realizar las tareas; reconocen y están de acuerdo con la existencia de los supervisores de zona; consideran que las decisiones son tomadas en forma participativa; en su mayoría se sienten valorados por la sociedad o población a la que asisten; concuerdan en decir que su labor tiene un alto grado de responsabilidad ya que de 


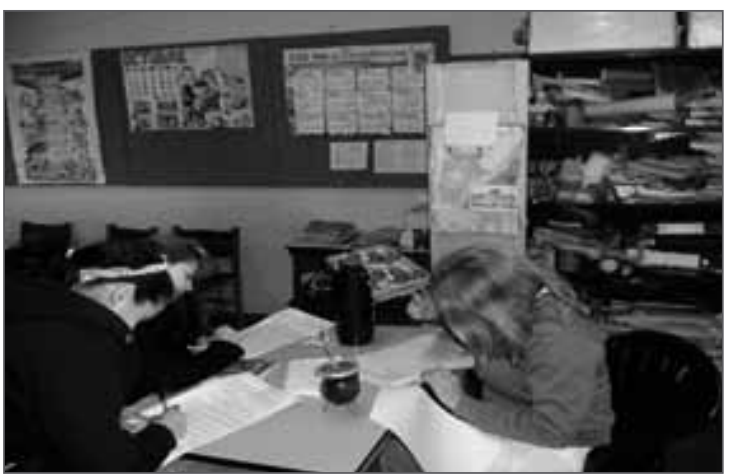

Figura 3: Entrevista a docentes rurales

ello depende el futuro de los niños en condiciones socio-económicas más vulnerables; están de acuerdo con las jornadas, licencias de trabajo y periodos de descanso, con las evaluaciones de desempeño que reciben y la comunicación que existe entre sus pares y superiores (directores, supervisores).

Los factores analizados nos estarían indicando que existen condiciones de trabajo óptimas, desde la perspectiva de la población estudiada y que, por ello, poseen una alta satisfacción laboral.

Asimismo, los aspectos considerados negativos están referidos a la percepción de inequidad entre el salario que perciben y las tareas que realizan; la ineficiencia del sistema previsional y de los beneficios sociales existentes; la ausencia de especialización para ejercer la docencia en zona rural; y la oferta de capacitación que reciben en cuanto a los contenidos y a la modalidad de las mismas, así como al hecho de que cuentan con un sistema de promociones que consideran injusto. Sin embargo, como punto "sobresaliente" coinciden en que la lejanía de sus lugares de trabajo dificulta la "comunicación” y por sobre todo "el cuidado de la salud de la población a la que asisten diariamente".

Éste último factor es resaltado como el de "mayor preocupación de éstos docentes".

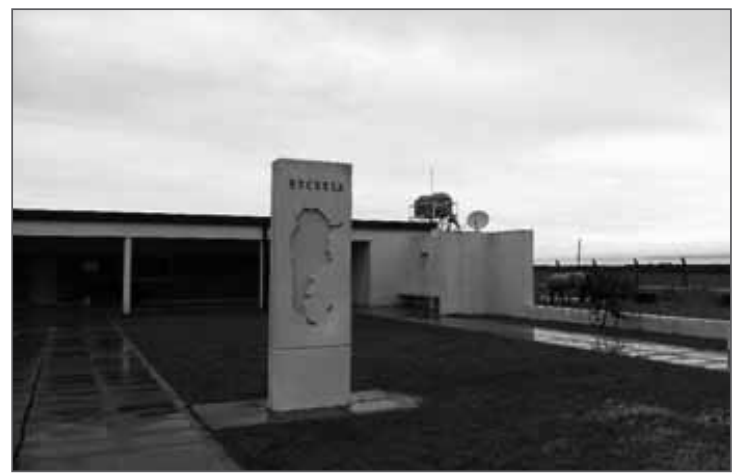

Figura 4. Visita a establecimientos educativos rurales

Toda ésta problemática provoca una carga emocional negativa, con riesgos psicosociales de éstos trabajadores, dato que fue confirmado por el gran número de casos de "licencias por estrés laboral y casos de depresión psicológica documentados".

Estos profesionales, están motivados e identificados por el contexto laboral tan particular en el que desempeñan sus actividades.

\section{Propuestas}

Del estudio se puede inferir que no sólo hay que tener en cuenta las condiciones internas y de entorno del trabajador, sino también la "percepción de bienestar del trabajador". Por ello se buscan alternativas de prevención de enfermedades de un sistema inmunitario menos eficiente (variación la concentración de glucosa y ácidos grasos, problemas cardiopulmonares, óseos, etc.), como la comunicación in-situ de profesionales de la salud y funcionarios, estimular la creatividad y cooperación, evitar la sensación de monotonía y aislamiento utilizando medios remotos.

Se propusieron a las autoridades del $\mathrm{Mi}^{-}$ nisterio de Educación de la provincia de Corrientes, acciones de trabajo que podrían aportar a las mejoras de las condiciones la- 
borales de los docentes y logar un mayor aprovechamiento de las Tecnologías de la Información:

a) Diseñar sistemas de capacitación especifica gratuita, destinadas a los docentes que ejercen la docencia en zona rural, con el objeto de brindar y otorgar las herramientas necesarias para atender a un sector particular de la población, y que sea adaptable en tiempo y espacio geográfico; todo ello a través de teleconferencias con los recursos existentes en los establecimientos (Internet).

b) Crear un sistema informático, destinado específicamente a mantener la comunicación directa entre autoridades del $\mathrm{Mi}^{-}$ nisterio de Educación y el personal docente que se encuentra en zonas desfavorables o alejadas de los organismos centrales. Un sistema apto para atender reclamos, inquietudes, sugerencias y cualquier otro tipo de información que requiera el docente ya que actualmente deben trasladarse desde su lugar de residencia hasta la ciudad de $\mathrm{Ca}^{-}$ pital para realizar trámites administrativos tales como solicitudes de certificaciones de
Ministerio puedan dirigirse directamente a cada uno de los docentes sin intermediarios que tienden en ocasiones, a distorsionar la comunicación.

c) Optimizar el área destinada al mejoramiento y control de la Higiene y Seguridad en el Trabajo, con el objeto eliminar posibles riesgos laborales y de atender la salud integral de los docentes. Toda ésta información, de existir un vínculo remoto, facilitaría el mantenimiento de las condiciones de seguridad e higiene.

d) Realizar visitas a los establecimientos educativos rurales que posean conectividad a internet, verificando el Ancho de banda BW disponible, para luego realizar las

pruebas necesarias de un sistema telemédico y su interrelación con los involucrados.

Para lograr mejores resultados en cuanto a la digitalización de los datos y su confidencialidad se propone desarrollar una plataforma a éste efecto, través de un portal de servicio denominado ceducom.net. Con el

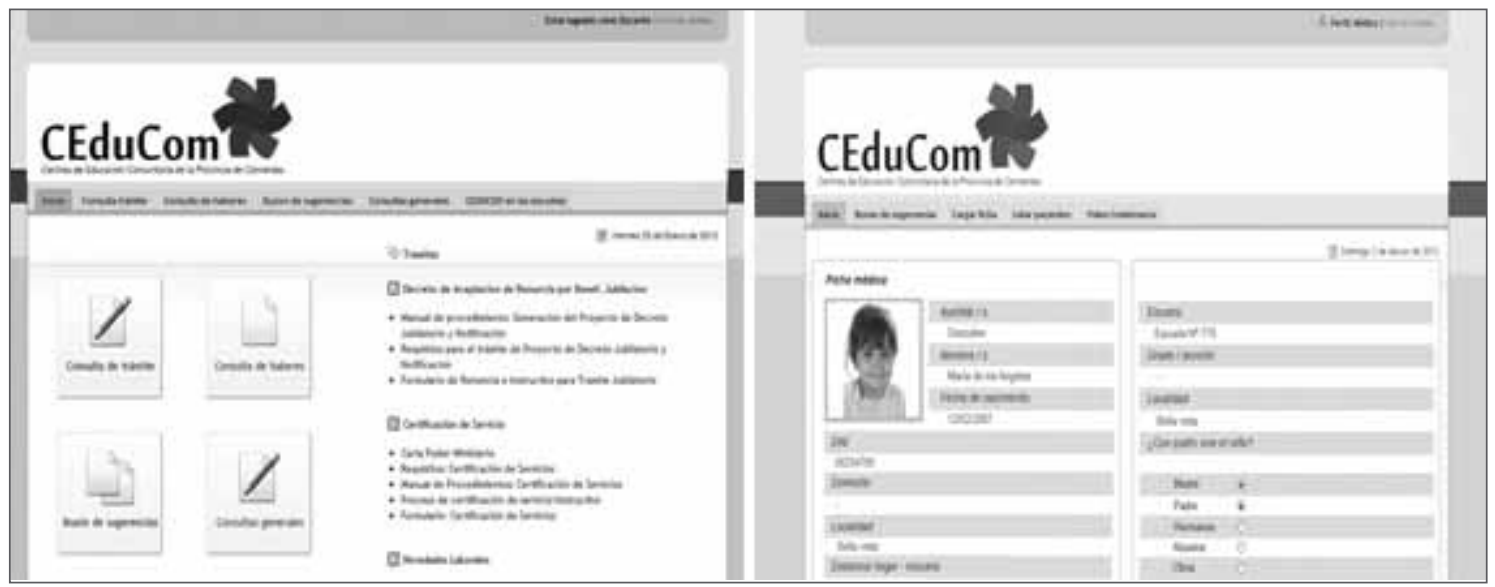

Figura 5. Portal de consulta de trámites administrativos para docentes

Figura 6. Ficha sanitaria digital (programa ceducom.net)

servicio, reclamos de conceptos de su salario, etc. Asimismo, que las autoridades del

objeto de atender la mayor preocupación detectada en el estudio efectuado en ésta 
población, consideramos imprescindible la generación de un área de Telemedicina Rural para los trabajadores docentes, pudiéndose extender el servicio a sus alumnos y familiares que radiquen en la zona del establecimiento. Idealmente, el sistema debe comprender el almacenamiento digital de fichas

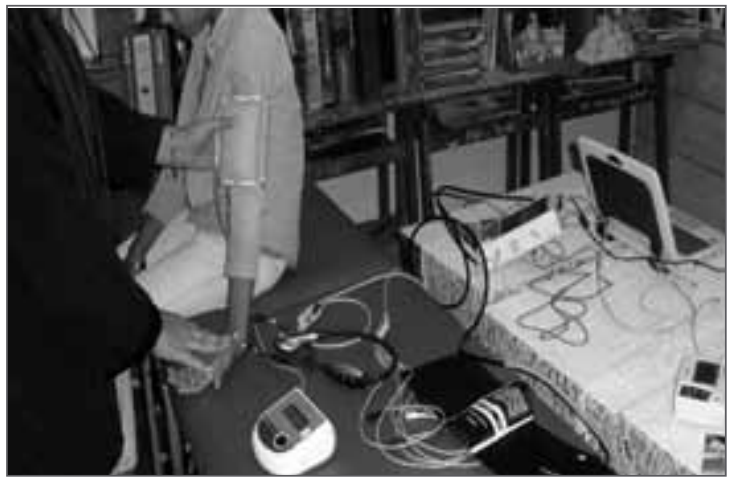

Figura 7. Captura de datos médicas, el uso de la TIC (videoconferencias, tele-consultas e interconsultas) y la incorporación de equipos de telemetría de parámetros médicos y colaborar en el desarrollo de programas de seguimiento, control y promoción de la salud de los involucrados.

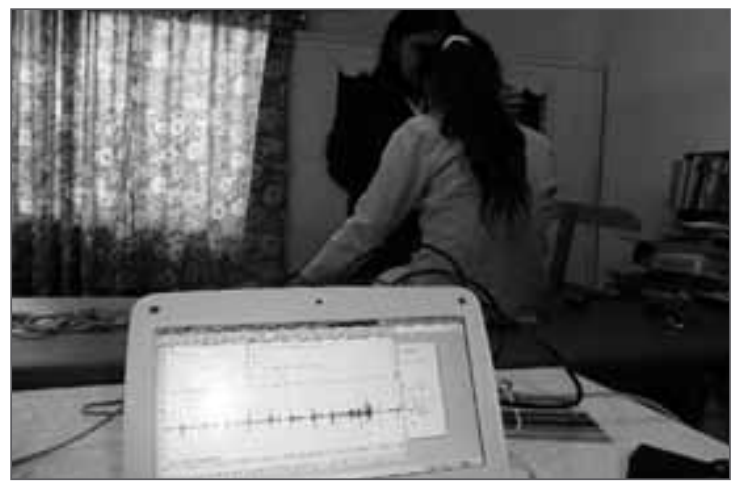

Figura 8. Carga de datos

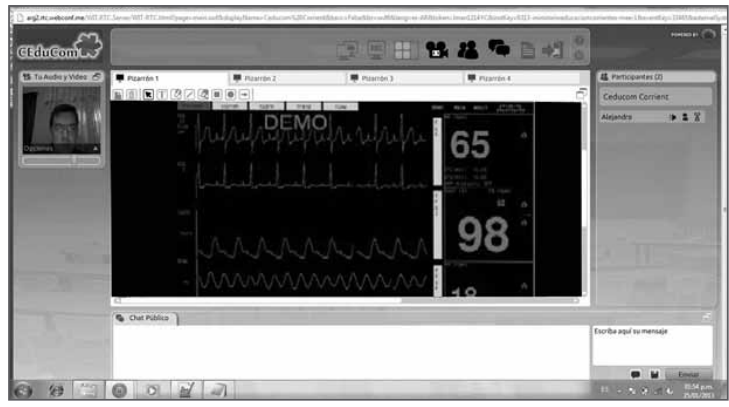

Figura 9. Recepción remota

\section{Bibliografía}

ABAD I, Carnicero J, (2012). Intercambio Internacional de Información Clínica. Manual de Salud Electrónica para directivos de servicios y sistemas de salud. ISBN: 978-84-695-2755-9, pp. 237-252.

ÁVILA DE TOMÁS, J F (2001). Aplicaciones de la telemedicina en atención primaria. Atención Primaria. Vol. 27, pp. 54-57.

BALDUCCI GONZÁLEZ, F. Hirigoyen
Emparanza G, Ramírez Payba CR, (2011). La instalación de equipos de telemedicina rural. Tecnología de la información y las comunicaciones para zonas rurales. ISBN: 978-84-15413-09-7, pp. 293-302.

BARREIRO MARTINS S, Simões, J, (2012). Aspectos éticos en el monitoreo remoto de marcapasos a través de la medicina. Revista Portuguesa de Bioética Vol. 16, pp. 51-54. 
BLANCO O, Rojas D, (2012). Principios de Seguridad de la Información en entornos de Salud. Manual de Salud Electrónica para directivos de servicios y sistemas de salud. ISBN: 978-84-695-2755-9, pp. 331-343.

CAICEDO RENDÓN OM, Corrales $\mathrm{Mu}^{-}$ ñoz JC, López Gutiérrez DM, Rendón Gallón A, (2011). Servicios web aplicados a la educación, la salud y el comercio. Tecnología de la información y las comunicaciones para zonas rurales. ISBN: 978-84-15413-09-7, pp. 63-82.

CANE LEÓN V, Hirigoyen Emparanza G, Belzarena García P J, (2011). Singularidades a tener en cuenta en un proyecto de telemedicina rural. Tecnología de la información y las comunicaciones para zonas rurales. ISBN: 978-84-15413-09-7, pp. 195-202.

CEUCLAENTE SILVA, J J (2004) Satisfacción en el Trabajo de los Directores de Escuelas Secundarias Públicas de la Región de Jacobina. Tesis Doctoral Facultad de Ciencias de la Educación. Bahia- Brasil.

FERRER ROCA O, (2001). Telemedicina, Editorial Panamericana. ISBN: 978-84-790-3606-5.

HOPPOCK, R (1935) Satisfacción en el Trabajo. Nueva York. Ed. Harper.

MARTÍNEZ, Deolidia (1990) El Riesgo de Enseñar. Secretaria de Cultura y Educación. Buenos Aires

MARTÍNEZ FERNÁNDEZ A, (2011). Evaluación del impacto de las TIC en la salud. Tecnología de la información y las comunicaciones para zonas rurales. ISBN: 978-84-15413-09-7, pp. 293-302.

NEFFA, J C (1988) ¿Qué son las condicio- nes y Medios ambiente de Trabajo? Propuesta de una nueva Perspectiva, Cap IV. CREDAL- Unidad asociada No 111 al Center Nacional de la Recherche Scientifique (CNRS) Editorial Hvmanitas.

NIETO, H (1999) Epidemiología de los accidentes de trabajo entre los trabajadores sanitarios. Rev. del Instituto de Higiene y Medicina Social.

NORRIS T, Hart G, Larson E, TarczyHornoch P, Masuda D, Fuller S, House P, Dyck S, (2002). Low-bandwidth, low-cost telemedicine consultations in rural family practice. J Am Board Fam Pract, Vol. 15, No. 2, pp. 123-127.

\section{ORGANIZACIÓN PANAMERICA-} NA DE LA SALUD (2003) Según cifras globales, en las Américas ocurren 36 accidentes de trabajo por minuto. Informe ESTRUCPLAN Satisfacción en el Trabajo. Nueva York. Ed. Harper ON LINE.

REY MORENO C, Liñán Benítez EL, Paco Fernández JA, Córdova Bernuy C, Quispe Tacas R, Simó Reigadas FJ, (2011). El diseño de la red de telecomunicación. Tecnología de la información y las comunicaciones para zonas rurales. ISBN: 978-84-15413-09-7, pp. 225-254.

RICUR G, (2012). Telemedicina: generalidades y áreas de aplicación. Manual de Salud Electrónica para directivos de servicios y sistemas de salud. ISBN: 978-84-695-2755-9, pp. 169-193.

SABINO, C (1996) El proceso de Investigación- Editorial Humanitas, Bs As.

VASILACHIS DE GIALDINO, I (2007) Estrategia de Investigación Cualitativo- Editorial Gedisa. Bs As. 